\title{
Some Result of Fuzzy Separation Axiom in Fuzzy Topological ring Space
}

\author{
Basim Mohammed Melgat ${ }^{1}$, Munir Abdul Khalik AL-Khafaji ${ }^{2}$ \\ ${ }^{I}$ Department of Public Health, College of Health and Medical Technique, Al-Furat Al-Awsat techniques / Kuffa \\ University \\ ${ }^{2}$ Department of Mathematics, College of Education, AL-Mustinsiryah University \\ ${ }^{*}$ Corresponding Author: Basimna73@Gmail.com \\ Received 18/11/2019, Accepted 28/11/2019, published 30/12/2012
}

DOI: $10.52113 / 2 / 06.02 .2019 / 13-19$

\begin{abstract}
In this paper, we study fuzzy separation axiom $T_{i}, i=0,1,2,3$ in the fuzzy topological ring space. Also the relationship between the types of fuzzy separation axiom was studied.

(C) 2019 Al Muthanna University. All rights reserved.
\end{abstract}

Keywords: Fuzzy topological ring; fuzzy $T_{i}$ space, $i=0,1,2,3$

\section{Introduction:}

In 1965 [11], Zadeh L. A. gave the definition of fuzziness. After three years $\mathrm{C}$. Chang [2] gave the notion of fuzzy topology. In 1990[1], Ahsanullah and Ganguli, depended on the convergent in fuzzy topological space in the sense of Lowen[7,8] to introduce the concept of fuzzy nbhd rings which gives the necessary and sufficient condition for a prefilter basis to be fuzzy nbhd prefilter of 0 in fuzzy topological ring. Also they are study the notions of right and left bounded fuzzy set and precompact fuzzy set fuzzy nbhd rings.

In 2009, Deb Ray, A. and Chettri, P [3] introduced fuzzy topology on a ring. Also in [4] they introduced fuzzy continuous function and studied left fuzzy topological ring. Our working to study fuzzy separation axiom $T_{i}, i=$ $0,1,2,3$ in the fuzzy topological ring space and obtaining the relationship between $T_{i}, i=$ $0,1,2,3$ spaces in the fuzzy topological ring space

For rich the paper, some basic concept of fuzzy set , fuzzy topology and fuzzy topological ring are given below. The symbol $I$ will denote to the closed interval $[0,1]$.

\subsection{Definition [11]}

A fuzzy set in $R$ is a map $\partial: R \rightarrow I$ and, that is, belonging to $I^{R}$ (the set of all fuzzy set of $R)$. Let $E \in I^{R}$, for every $r \in R$, we expressed by $E(r)$ of the degree of membership of $r$ in $R$. 
If $E(r)$ be an element of $\{0,1\}$, then $E$ is said a crisp set.

\subsection{Definition [2]}

A class $\mu \in I^{R}$ of fuzzy set is called a fuzzy topology for $R$ if the following are satisfied

1) $\emptyset, R \in \mu$

2) $\forall E, H \in \mu \rightarrow E \wedge H \in \mu$

3) $\forall\left(E_{j}\right)_{j \in J} \in \mu \rightarrow \vee_{j \in J} E_{j} \in \mu$

$(R, \mu)$ is called fuzzy topological space. if $A \in \mu$ Then $A$ is fuzzy open and $A^{c}$ (complement of $A$ ) is a fuzzy closed set.

\subsection{Definition $[1,3]$}

A pair $(R, \mu)$, where $R$ a ring and $\mu$ be a fuzzy topology on $R$, is called fuzzy topological ring if the following maps are fuzzy continuous:

1) $R \times R \rightarrow R,(r, k) \rightarrow r+k$.

2) $R \rightarrow R, r \rightarrow-r$

3) $R \times R \rightarrow R,(r, k) \rightarrow r . k$

\subsection{Definition [4]}

A family $B$ of fuzzy nbhds of $r_{\alpha}$, for $0<$ $\alpha \leq 1$, is called a fund. system of fuzzy nbhds of $r_{\alpha}$ iff for any fuzzy nbhd $V$ of $r_{\alpha}$, there is $U \in$ $B$ such that $r_{\alpha} \leq U \leq V$

\subsection{Definition [4]}

Let $R$ be a ring and $\mu$ a fuzzy topological on $R$. Let $U$ and $V$ are fuzzy sets in $R$. We define $U+V,-V$ and $U . V$ as follows

$$
\begin{aligned}
& (U+V)(k)=\sup _{k=k_{1}+k_{2}} \min \left\{U\left(k_{1}\right), V\left(k_{2}\right)\right\} \\
& -V(k)=V(-k) \\
& (U . V)(k)=\sup _{k=k_{1}+k_{2}} \min \left\{U\left(k_{1}\right), V\left(k_{2}\right)\right\}
\end{aligned}
$$

\subsection{Theorem [4]}

If $R$ is a fuzzy topological ring then there is a fundamental system of fuzzy nbhds $B$ of 0 $(0<\alpha \leq 1)$, such that the conditions:

(i) $\forall U \in B$, then $-U \in B$

(ii) $\forall U \in B$, then $U$ is symmetric

(iii) $\forall U, V \in B$, then $U \wedge V \in B$

(iv) $\forall U \in B$, there is $V \in B$ such that $V+V \leq$ $U$

(v) $\forall U \in B$, there is $V \in B$ such that $V . V \leq U$

(vi) $\forall r \in R, \forall U \in B$, there is $V \in B$ such that a $r . V \leq U$ and $V . r \leq U$.

\subsection{Definition [7]}

$(R, \mu)$ is fully stratified fuzzy topology on $R$ if the fuzzy topology $\mu$ on $R$ contain all constant fuzzy set

\subsection{Definition [10]}

A fuzzy topological space $(R, \mu)$ is said to be fuzzy $T_{0}$-topological space iff $\forall r_{\alpha}, k_{\alpha} \in$ $R, r \neq k, \exists U \in \mu$ such that either $U(r)=1$ and $U(k)=0$ or $U(k)=1$ and $U(r)=0$.

\subsection{Definition [10]}

A fuzzy topological space $(R, \mu)$ is said to be fuzzy $T_{1}$ - topological space iff $\forall r_{\alpha}, k_{\alpha} \in R, r \neq k, \exists U, V \in \mu$ such that 


$$
\begin{aligned}
& U(r)=1, U(k)=0 \text { and } V(r)=0, \\
& V(k)=1
\end{aligned}
$$

\subsection{Definition [10]}

A fuzzy topological space $(R, \mu)$ is said to be fuzzy Hausdorff or fuzzy $T_{2}$ space iff for any two distinct fuzzy points $r_{\alpha}, k_{\alpha} \in R$, there exists disjoint fuzzy sets $U, V \in \mu$ with

$$
U(r)=V(k)=1 .
$$

\subsection{Definition [10]}

A fuzzy topological space

$(R, \mu)$ will be called fuzzy regular if for each fuzzy point $r_{\alpha}$ and each fuzzy closed set $H$ such that $H(r)=0$ there are fuzzy open sets $U$ and $V$ such that $U(r)>0, H \leq V$ and $U \wedge V=\emptyset$.

\subsection{Proposition [10]}

If a space $R$ is a fuzzy regular space, then for any open set $U$ and a fuzzy point $r_{\alpha} \in R$ such that $c l(U)(r)=0$ there exists an open set $V$ such that $\alpha \leq V \leq \operatorname{cl}(V) \leq U$

\subsection{Definition [10]}

A fuzzy topological $\operatorname{space}(R, \mu)$ will be called normal if for each pair of fuzzy closed sets $H_{1}$ and $H_{2}$ such that $H_{1} \wedge H_{2}=\varnothing$ there exist fuzzy open sets $U_{1}$ and $U_{2}$ such that $U_{1} \leq H_{1}$ and $U_{2} \leq H_{2}$ and $U_{1} \wedge U_{2}=\varnothing$.

\subsection{Definition [10]}

\section{A fuzzy topological}

space $(R, \mu)$ is said to be fuzzy

$T_{3}$-topological space iff it is

fuzzy $T_{1}$-and fuzzy regular.

\section{Separation Axiom}

\subsection{Theorem}

Let $(R, \mu)$ be a fuzzy topological ring. If $k_{\alpha} \in\left\{r_{\alpha}: R(r)=\max \{R(h)\}, \forall h \in R\right\}$ and $U$ is a fuzzy nbhd of 0 , then $k+U$ is a fuzzy nbhd of $k$ such that $(k+U)(k)=\max \{R(h)\}$, $\left.\forall h_{\alpha} \in R\right\}$.

Proof

Since $U$ is a fuzzy nbhd of 0 , there exists $V$ fuzzy open set such that $V \subseteq U$ and $V(0)=U(0)=\max \{R(h)\}, \forall h \in R\}$. Let $g_{k}\left(r_{\alpha}\right):(R, \mu) \rightarrow(R, \mu), g_{k}(r)=r_{\alpha}+k_{\alpha} \cdot g_{k}$ is a fuzzy homeo. Thus $k+V$ is a fuzzy open set.

$$
\begin{gathered}
k+V(k)=V(k-k)=V(0) \\
=\max \{R(h)\}, \quad \forall h \in R . \\
k+U(r)=U(r-k) \geq V(r-k)=k+ \\
V(r) \text { for all } r \in R .
\end{gathered}
$$

Thus there exists $k+V$ fuzzy open such that $k+V \leq k+U$ and $(k+V)(K)=(k+$ $U)(k)=\max \{R(h)\}, \forall h \in R$

\subsection{Theorem}

Let $(R, \mu)$ be a fuzzy topological ring. If $k_{\alpha} \in\left\{r_{\alpha}: R(r)=\max \{R(h)\}, \forall h \in R\right\}$ and 
$U$ is a fuzzy nbhd of $k_{\alpha}$ such that $U(k)=$ $\max \{R(h)\}, \forall h \in R$, then $U-k$ is a fuzzy nbhd of 0 such that $(U)(0)=\max \{R(h)\}$, $\forall h \in R$.

\section{Proof}

Since $U$ is a fuzzy nbhd of $k_{\alpha}$, there exists $V$ fuzzy open set such that $V \subseteq U$ and $V(k)=U(k)=\max \{R(h)\}, \forall h \in R$. Let $g_{k}: R \rightarrow R, g_{k}\left(r_{\alpha}\right)=r_{\alpha}-k_{\alpha}, g_{k}$ is a fuzzy homeo. Thus $-k+V$ is a fuzzy open set.

$V-k(0)=V(0+k)=V(k)=\max \{R(h)\}$, $\forall h \in R\}=V(0)$.

$U-k(r)=U(r+k) \geq V(r+k)=V-$ $k(r)$ for all $r \in R$.

Thus there exists $V-k$ fuzzy open set such that $V-k \leq U-k$ and $V(0)=U-k(0)=$ $\max \{R(h)\}, \forall h \in R\}$.

\subsection{Definition}

A fuzzy topological ring $(R, \mu)$ is said to be Fuzzy $\mathrm{T}_{0}$ - space iff for each $\mathrm{r}_{\alpha}, \mathrm{k}_{\alpha}$ s.t $\mathrm{r} \neq \mathrm{k}$ there exists fuzzy open set $U$ s.t $\mathrm{r}_{\alpha} \in U, \mathrm{k}_{\alpha} \notin U$ or $\mathrm{k}_{\alpha} \in U, \mathrm{r}_{\alpha} \notin U$

\subsection{Example}

Let $\mathrm{Z}_{2}$ be the ring of integers

modulo 2. Define fuzzy sets $E_{1}, E_{2}, E_{3}$ on $\mathrm{Z}_{2}$ as

$$
\begin{aligned}
& E_{1}([0])=0.9, E_{1}([1])=0, \\
& E_{2}([0])=0, E_{2}([1])=0.9
\end{aligned}
$$

for all $r \in \mathrm{Z}_{2}$. Let $\mu=\left\{\varnothing, \mathrm{Z}_{2}, E_{1}, E_{2}\right\}$ is a fuzzy topological ring on $Z_{2}$, then $\left(Z_{2}, \mu\right)$ is a fuzzy $\mathrm{T}_{0}$ - topological ring space.

\subsection{Definition}

A fuzzy topological ring $(R, \mu)$ is said to be Fuzzy $\mathrm{T}_{1}$ - space iff for each $\mathrm{r}_{\alpha}, \mathrm{k}_{\alpha}$ s.t $\mathrm{r} \neq \mathrm{k}$ there exists fuzzy open sets $U, V$ s.t $\mathrm{r}_{\alpha} \in$ $U, \mathrm{k}_{\alpha} \notin U$ and $\mathrm{k}_{\alpha} \in V, \mathrm{r}_{\alpha} \notin V$

\subsection{Example}

Let $\mathrm{Z}_{2}$ be the ring of integers

modulo 2. Define fuzzy sets $E_{1}, E_{2}, E_{3}$ on $\mathrm{Z}_{2}$ as $E_{1}([0])=0.25, E_{1}([1])=0$, $E_{2}([0])=0, E_{2}([1])=0.25$ $E_{3}([0])=0.25, E_{3}([1])=0.25$ for all $r \in \mathrm{Z}_{2}$. Let $\mu=\left\{\varnothing, \mathrm{Z}_{2}, E_{1}, E_{2}, E_{3}\right\}$ is a fuzzy topological ring on $Z_{2}$, then $\left(Z_{2}, \mu\right)$ is a fuzzy $\mathrm{T}_{1}$ - topological ring space.

\subsection{Definition}

A fuzzy topological ring $(R, \mu)$ is said to be Fuzzy $\mathrm{T}_{2}$ - topological ring space iff for any two fuzzy points $\mathrm{r}_{\alpha}, \mathrm{k}_{\beta} \quad$ s.t $\mathrm{r} \notin \operatorname{supp}(\mathrm{k})$ and $k \notin$ $\operatorname{supp}(r)$, there exists two fuzzy open sets $\mathrm{U}, \mathrm{V}$ s.t $\mathrm{r}_{\alpha} \in U, \mathrm{k}_{\alpha} \in V$ and $U \wedge V=\varnothing$

\subsection{Example}

Let $\mathrm{Z}_{4}$ be the ring of integers

modulo 4 , with fuzzy discrete topology $\mu_{D}$ on it. The fuzzy topological ring $\left(\mathrm{Z}_{4}, \mu_{D}\right)$ is Fuzzy $\mathrm{T}_{2}$ - topological ring space.

\subsection{Definition}

A fuzzy topological ring $(R, \mu)$ is said to be Fuzzy regular space if $\forall r_{\alpha} \in R$ and a fuzzy closed set $F$ with $F(r)=0$ there exists 
fuzzy open sets $\mathrm{U}, \mathrm{V}$ such that $r_{\alpha} \in U$ and $F \subset$ $V$ and $\wedge V=\varnothing$.

\subsection{Definition}

A fuzzy topological ring $(R, \mu)$ is said to be Fuzzy $T_{3}$-topological ring space if $(R, \mu)$ is fuzzy $T_{1}$ - space and fuzzy regular topological ring space.

\subsection{Example}

Let $\mathbb{R}$ be the ring of real number, with fuzzy usual topology $\mu_{U}$ on it. Then $\left(\mathbb{R}, \mu_{U}\right)$ is Fuzzy $\mathrm{T}_{3}$ - topological ring space

\subsection{Theorem}

If $(R, \mu)$ is fuzzy $T_{2}$ - topological ring space then $\left\{0_{\alpha}\right\}$ is fuzzy closed subset in $(R, \mu)$.

\section{Proof}

Let $(R, \mu)$ is fuzzy $T_{2}$ - topological ring space. For any $r_{\alpha} \neq 0_{\alpha}$ be another fuzzy point, assume $U(r)>0, \forall U \in\left\{B_{0}\right\}$, then there exists fuzzy open set $V$ of $r_{\alpha}$ s.t $V(0)=0$, implies $\overline{\{0\}}(r)=0$. Since $r$ is arbitrary. Thus $\overline{\left\{0_{\alpha}\right\}}=\left\{0_{\alpha}\right\}$ and $\left\{0_{\alpha}\right\}$ is fuzzy closed set.

\subsection{Theorem}

For any fuzzy topological ring space $(R, \mu)$, if $\left\{0_{\alpha}\right\}$ is fuzzy closed subset in $R$ and if $B_{0}$ is a basis of fuzzy nbhd of $0_{\alpha}$, then $\wedge_{V \in\left\{B_{0}\right\}} V=\left\{0_{\alpha}\right\}$.

proof
Let $\overline{\left\{0_{\alpha}\right\}}$ be an fuzzy closed set and $\left\{B_{0}\right\}$ be a basis of fuzzy nbhds of $0_{\alpha}$. Then by theorem 2.12, $\left\{0_{\alpha}\right\}=\overline{\left\{0_{\alpha}\right\}}=\Lambda_{V \in\left\{B_{0}\right\}}\left(\left\{0_{\alpha}\right\}+V\right)=\Lambda_{V \in\left\{B_{0}\right\}} V$

Thus $\bigwedge_{V \in\left\{B_{0}\right\}} V=\left\{0_{\alpha}\right\}$

\subsection{Theorem}

For any fuzzy topological ring space $(R, \mu)$, and $B_{0}$ is a basis of fuzzy nbhd of $0_{\alpha}$, if $\wedge_{V \in\left\{B_{0}\right\}} V=$ $\left\{0_{\alpha}\right\}$, then $(\mathrm{R}, \mu)$ is fuzzy $T_{0}$-topological ring space.

\section{Proof}

Let $\left\{B_{0}\right\}$ be a basis of fuzzy nbhds of $0_{\alpha}$ and $\bigwedge_{V \in\left\{B_{0}\right\}} V=\left\{0_{\alpha}\right\}$. Let $r_{\alpha}$ and $k_{\alpha}$ are fuzzy points with different support. Therefor $r_{\alpha}-k_{\alpha} \neq 0$, so there exists $V \in\left\{B_{0}\right\}$, s.t $V(r-k)=0$. Now by theorem 1.6, $k+V(r)$ is fuzzy nbhd of $k_{\alpha}$ and $(k+V)(r)=V(r-k)=0$. Thus $r_{\alpha} \notin k+V$ and $(R, \mu)$ is fuzzy $T_{0}$-topological ring space.

\subsection{Theorem}

If $(R, \mu)$ is fuzzy $T_{0^{-}}$topological ring space, Then $(R, \mu)$ is fuzzy $T_{1}$-topological ring space

\section{Proof}

Let $(\mathrm{R}, \mu)$ be a fuzzy $T_{0}$-topological ring space and let $r_{\alpha}$ and $k_{\alpha}$ are fuzzy points with different support. Then there exists fuzzy open set $V$ of $0_{\alpha}$ s.t $(r+V)(k)=0$ or $(k+V)(r)=0$. We can assume that $V$ is symmetric fuzzy open set of $0_{\alpha}$. To explain that $(r+V)(k)=0$ if $(k+$ 
$V)(r)=0$ we assume the contrary, suppose For any fuzzy topological ring $(R, \mu)$, the that $(r+V)(k)>0, \quad$ therefor $(r-V)(k)>0$. following conditions are equivalent

Implies

$(r+V)(k)=V(k-r)=V(-(r-k))=$

$V(r-K)=(k+V)(r)>0$.

This contradiction, similarly if $(k+V)(r)=0$. Thus $(\mathrm{R}, \mu)$ is fuzzy $T_{1}$-topological ring space.
1) $(R, \mu)$ is fuzzy $T_{2}$-topological ring space

2) $\left\{0_{\alpha}\right\}$ is FZ.closed subset in $R$.

3) If $B_{0}$ is a basis of nbhd of $0_{\alpha}$, then $\bigcap_{V \in B_{0}} V=$ $\left\{0_{\alpha}\right\}$

4) $(R, \mu)$ is fuzzy $T_{0}$-topological ring space.

5) $(R, \mu)$ is fuzzy $T_{1}$-topological ring space.

6) $(R, \mu)$ is fuzzy $T_{3}$-topological ring space.

\section{Proof}

By theorems 2.10, 2.11, 2.12, 2.13 and 2.14

\subsection{Theorem}

If $(R, \mu)$ is fuzzy $T_{1}$-topological ring space then $(R, \mu)$ is fuzzy $T_{3}$-topological ring space.

\section{Proof}

Let $(R, \mu)$ is fuzzy $T_{1}$-topological ring space and let $r \in R, F$ be a fuzzy closed subset in $R$ s.t $F(r)=0$, then $F^{c}(r)=1$ and $F^{c}$ is fuzzy open set. Therefor $F^{c}-r$ is an fuzzy open nbhd system of $0_{\alpha}$. Then there exists fuzzy open set $V_{0}$ of $0_{\alpha}$ s.t $V_{0} \leq F^{c}-r$. Now

$\overline{V_{0}}=\bigcap\left(V_{0}+\left(F^{c}-r\right)\right)=\bigcap\left(F^{c}-r\right)=\left\{0_{\alpha}\right\}$,

$\left(r+\overline{V_{0}}\right)(k)=\overline{V_{0}}(k-r)=\min \left\{\overline{V_{0}}(k), \overline{V_{0}}(-r)\right\}=$ $\min \left\{\overline{V_{0}}(k), \overline{V_{0}}(r)\right\}=\bar{V}_{0}(k)=V_{0}(k) \quad, \forall k \in R$

implies $r+\overline{V_{0}} \leq F^{c}-r$. Thus $(\mathrm{R}, \mu)$ is fuzzy regular and consequently it is fuzzy $T_{3}$-topological ring space.

\subsection{Theorem}

\subsection{Theorem}

Let $(R, \mu)$ be a fuzzy topological ring, then Every fuzzy subspace of fuzzy $T_{0}$-space is a fuzzy $T_{0}-$ space.

\section{Proof:}

Obvious

\subsection{Theorem}

$\operatorname{Let}(R, \mu)$ be a fuzzy topological ring and $E$ is a fuzzy subring of $(R, \mu)$, if $\left(E, \mu_{E}\right)$ is fuzzy Hausdorff and $(R / E, \beta)$ is fuzzy Hausdorff then $(R, \mu)$ is fuzzy Hausdorff.

\section{Proof}

If $R / E$ is fuzzy Hausdorff, then $E$ is fuzzy closed set in $(R, \mu)$.

If also $\left(E, \mu_{E}\right)$ is fuzzy Hausdorff then $\left\{0_{\alpha}\right\}$ is fuzzy closed in $E$.

Hence $\left\{0_{\alpha}\right\}$ is fuzzy closed in $(R, \mu)$, and $(R, \mu)$ is fuzzy Hausdorff. 


\section{References}

[1] Ahsanullah T. M. G., On Fuzzy

Neighborhood Ring, Fuzzy Set and Systems

34(1990) 255-262 North Holland

[2] Chang, C. L : Fuzzy topological spaces.

Math. Anal. Appl.,24(1968),182-190.

[3] Deb Ray, A and Chettri, P: On Fuzzy

Topological Ring Valued Fuzzy Continuous

Functions "Applied Mathematical Sciences, 2009, Vol. 3, no. 24, 1177 - 1188

[4] Deb Ray, A : On (left) fuzzy topological ring. Int. Math , (2011), vol. 6, no. 25 -

$28,1303-1312$.

[5] Das, N.R. and Das, P, (2000), Neighborhood systems in fuzzy topological groups. Fuzzy

Sets and Systems, 116 401-408

[6] R. Lowen, Fuzzy topological spaces and fuzzy compactness, J. Math.

Anal. Appl, 56(1976) 621-633

[7] Lowen R., Convergence in a fuzzy

topological space, Gen. Topology

Appl. 10 (1979)147-160

[8] Lowen R., Fuzzy neighborhood spaces, J.

Fuzzy Sets and Systems

7(1982), 165-189

[9] Palaniappan N., Fuzzy topology, Narosa

Publications, 2002.

[10] Warner S.: Topological Rings, North-

Holland Math. 1993

[11] Zadeh, L.A : Fuzzy Sets, Information and

Control,8(1965), 338-353. 